\title{
"Cartas desde nuestro corazón": una experiencia de aprendizaje-servicio para favorecer la inclusión de las personas con enfermedades raras
}

\author{
Berta Paz \\ Sebastià Verger \\ Universitat de les Illes Balears, España \\ Asociación INèDITHOS, España \\ Kate Brennan \\ Asociación INèDITHOS, España
}

\section{Resumen}

El proyecto fue desarrollado en el curso 2018-19 entre la asociación INèDITHOS y el centro educativo Escola Global, y tuvo como objetivo facilitar la comunicación a través de cartas entre estudiantes y niños con enfermedades raras, además de otras acciones vinculadas a la sensibilización y la captación de fondos para INèDITHOS, una entidad que desarrolla soporte educativo y lúdico a niños en el hospital y domicilio.

\section{Palabras clave}

Enfermedades crónicas, escuela, inclusión. 


\title{
"Letters from the heart": a service-learning experience to foster the inclusion of people with rare diseases
}

\begin{abstract}
This paper describes a project developed in the 2018-19 academic year between the INèDITHOS association and the Escola Global school, with the aim of facilitating communication through letters between students and children with rare diseases, in addition to other actions aimed at raising awareness and raising funds for INèDITHOS, an entity that offers educational and recreational support to sick children in hospital and at home.
\end{abstract}

\section{Keywords}

Chronic diseases, school, inclusion 


\section{Marco del proyecto}

Este proyecto se desarrolló en Mallorca, España, e implicó a la asociación INèDITHOS y al centro educativo Escola Global durante el curso 2018-19. Escola Global es un colegio internacional privado creado en 2008 y ubicado en el parque tecnológico denominado Parc Bit, en Palma. Se trata de un colegio de pequeño tamaño, que sigue el currículum británico y está también homologado por la Consejería de Educación de las Islas Baleares. Es conocido por el desarrollo de metodologías activas como aprendizaje por problemas o aprendizaje por proyectos y cuenta con una amplia trayectoria de colaboración con asociaciones y entidades benéficas. Sin embargo, no había articulado ningún proyecto específicamente diseñado y evaluado como aprendizaje-servicio hasta la fecha. Estos proyectos tienen unas características concretas y sus beneficios han sido ampliamente estudiados (Eyler et al., 1997; Puig et al., 2008; Rubio, 2009; Lake y Jones, 2012).

Por su parte, la organización INèDITHOS, que había surgido en 2003 para dar un soporte educativo y lúdico a niños que vivían permanentemente en la unidad pediátrica de semicríticos del Hospital Son Espases (Palma, Islas Baleares, España), continúa hoy atendiendo a niños del hospital, pero también ofrece soporte domiciliario a miembros de ABAIMAR (Asociación Balear de niños con Enfermedades Raras). La entidad ha sido premiada a nivel local, nacional e internacional por su papel en lo que se denomina pedagogía hospitalaria (Chaves, 2012). Esta asociación sí tenía experiencia en la participación en proyectos de aprendizaje-servicio con alumnos universitarios, siendo entidad contraparte en proyectos de Innovación docente de la Universitat de les Illes Balears, así como de proyectos de investigación educativa financiados a nivel nacional. En este caso, no contaba con experiencias a largo plazo en la que el servicio fuera ofrecido específicamente por niños de edades similares a la de los niños enfermos que la asociación atiende. Para el desarrollo de este proyecto se realizaron varias reuniones previas a fin de determinar aquellas necesidades de INèDITHOS que pudieran suponer una fuente de aprendizaje para el alumnado de Escola Global.

Así, surge toda una propuesta de aprendizaje-servicio consensuada con ambas partes, que colaboran en el diseño desde el inicio, tal y como se recomienda en la literatura (Puig et al., 2008). La creación de material educativo fue uno de los productos de servicio comentados en las reuniones previas, tal vez en forma de cuentos, dibujos, etc. Sin embargo, se decide empezar por un intercambio de cartas, entendiendo que con ello se podría trabajar también otros aspectos relacionados con la adquisición de valores, el espíritu crítico y la sensibilización sobre otras realidades que tienen los niños en un entorno cercano. Así, surge el inicio de una actividad de aprendizaje-servicio que englobó a ambas organizaciones con diferentes actividades a lo largo del curso escolar con un papel primordial

Paz, B.; Brennan, K. y Verger, S. (2021). Cartas desde nuestro corazón: una experiencia de aprendizajeservicio para favorecer la inclusión de las personas con enfermedades raras. RIDAS, Revista Iberoamericana de Aprendizaje Servicio, 11, 117-127. DOI10.1344/RIDAS2021.11.8 
del alumnado y de los niños y niñas que reciben soporte educativo de INèDITHOS. De esta manera, se articula un trabajo en red que implica al profesorado del centro, al alumnado, a la asociación de padres y madres, a la organización y voluntarios de INèDITHOS, a los niños y las familias, muchos de ellos miembros de ABAIMAR.

El eje vertebrador de este proyecto titulado "Cartas desde nuestro corazón" supone el aprendizaje en las áreas de lectoescritura para alumnos de primaria y secundaria que realizan un servicio de apoyo con recursos educativos (cartas) y sociales a la organización de la asociación INèDITHOS. Se establece así una comunicación directa entre los niños con enfermedades crónicas y raras a los que esta asociación atiende en el entorno hospitalario y domiciliario.

\section{Sinopsis del proyecto}

\section{Este proyecto se vincula}

fundamentalmente con tres objetivos de desarrollo sostenible. El objetivo tres que se refiere a la salud y bienestar, el objetivo cuatro sobre educación de calidad y el objetivo diecisiete, referente a alianzas para lograr objetivos.

Este proyecto aprendizaje-servicio permite que niños de primaria y secundaria escriban cartas a niños con enfermedades raras y crónicas que viven en la unidad de semicríticos del hospital Son Espases (Hospital de referencia en Baleares) a los que da soporte lúdico y educativo la asociación INèDITHOS. Ello permite, además del desarrollo de competencias de lecto- escritura, la sensibilización de los menores hacia otros niños que están enfermos, así como el conocimiento de la realidad que viven los niños con enfermedades raras y sus familias (Verger et al., 2017). Por su parte, para INèDITHOS, las cartas son un servicio que permite trabajar con los niños y niñas hospitalizados o en el domicilio también las competencias de lectoescritura, pero sobre todo permite también un apoyo emocional, un soporte entre iguales y una inclusión a una vida más normalizada para estos niños, que en algunos casos están privados del ambiente socializador que permite la escuela. Por lo tanto, en ambos casos se busca un aprendizaje significativo, pues el texto escrito va dirigido otra persona que responderá en unas semanas. La primera actividad de intercambio de cartas tiene lugar el día 14 de febrero, lo que permitió romper también con la dinámica consumista de este día particular para profundizar en la sensibilización y apoyo a los grupos sociales que más lo necesitan. Además de las cartas elaboradas por los alumnos, el colegio donó material fungible y escolar para los niños. Este primer momento de entrega de cartas desde Escola Global a la asociación INèDITHOS fue grabado y emitido por la televisión de carácter autonómico Canal 4, refiriéndose al mismo como un ejemplo de solidaridad.

Por su parte, miembros y voluntarios de INèDITHOS también registraron el proceso de entrega de las cartas a los niños atendidos en la asociación, respetando su anonimato cuando así lo deseaban las familias o mostrándolos cuando se contaba con el

Paz, B.; Brennan, K. y Verger, S. (2021). Cartas desde nuestro corazón: una experiencia de aprendizajeservicio para favorecer la inclusión de las personas con enfermedades raras. RIDAS, Revista Iberoamericana de Aprendizaje Servicio, 11, 117-127. DOI10.1344/RIDAS2021.11.8 
consentimiento de los padres para su participación en la grabación. Este material audiovisual se completó con información sobre la asociación y se preparó un vídeo que posteriormente se emitió en todas las clases del colegio, junto con la participación de tres miembros de INèDITHOS. Ello sirvió para aportar más información sobre la organización, para mostrar la recepción de las cartas por parte de los niños, el trabajo de los niños para dar respuesta a las cartas y también para sensibilizar al alumnado sobre las enfermedades raras. Adicionalmente a la redacción de las cartas se llevan a cabo cuatro estrategias de recogida de fondos organizadas por el alumnado de secundaria, el centro escolar y la Asociación de padres y madres del centro.

\section{Necesidad social que atiende el proyecto}

Las siglas INèDITHOS significan "Intervención e Investigación para la inclusión educativa y tecnología en Pedagogía Hospitalaria" (Negre y Verger, 2005). En su página web $^{1}$ se explica el funcionamiento $y$ características de la intervención, así como su relación con investigación y la innovación docente que hasta la fecha desarrollaba fundamentalmente en el ámbito universitario. En la actualidad gestiona a más de cincuenta voluntarios, principalmente estudiantes de Grado de Educación Infantil, Pedagogía, Educación Primaria, Psicología, Biología, Derecho, etc. No es infrecuente que muchos de estos alumnos opten por realizar proyectos de aprendizaje-servicio en sus asignaturas de grado, así como Doctorado en Educación y Máster de Educación Inclusiva. Si bien la trayectoria de INèDITHOS tiene su inicio a comienzos del 2000, se constituye como una asociación sin ánimo de lucro en el año 2016 inscrita con el número 311000009645 de la Sección Primera del Registro de Asociaciones de las Islas Baleares.

Por ello, la necesidad social que atiende este proyecto tiene que ver con las propias funciones de INèDITHOS, que es una asociación dirigida a la mejora de la calidad de vida de niños y jóvenes en situación de enfermedad (especialmente enfermedades crónicas y/o enfermedades raras) y a sus familiares. En concreto se ejecuta un programa educativo y de apoyo psicopedagógico en la Unidad de Semicríticos del Hospital Son Espases (hospital público de referencia en Baleares) atendiendo a pacientes cuya enfermedad les obliga a residir, de forma permanente en esta Unidad de Cuidados Intensivos (Ortiz et al., 2017). También desarrolla refuerzo domiciliario basada en las necesidades educativas de niños y jóvenes con enfermedades poco frecuentes (Fernández y Grau, 2014) y a pacientes pediátricos a la Unidad de Cuidados Paliativos del Hospital Sant Joan de Déu de Palma de Mallorca.

En concreto, las necesidades a las que atiende el proyecto son de cuatro tipos:

a) Aporta material didáctico para el voluntariado, los niños y niñas y sus familias

\footnotetext{
${ }^{1}$ www.inedithos.es
}

Paz, B.; Brennan, K. y Verger, S. (2021). Cartas desde nuestro corazón: una experiencia de aprendizajeservicio para favorecer la inclusión de las personas con enfermedades raras. RIDAS, Revista Iberoamericana de Aprendizaje Servicio, 11, 117-127. DOI10.1344/RIDAS2021.11.8 
b) Presta apoyo emocional y sentido de pertenencia al grupo para los niños enfermos

c) Aporta solvencia financiera para la adquisición de material didáctico y tecnológico

d) Colabora en la sensibilización de la sociedad sobre la situación de los niños con enfermedades crónicas y raras con necesidades educativas y lúdicas.

En su corta trayectoria como asociación ha contado con varios reconocimientos, destacando el PREMIO FEDER

(Federación Española de Enfermedades Raras) a la Inclusión, el premio más prestigioso a nivel nacional específicamente referido a las entidades que colaboran con personas afectadas de enfermedades raras, 2017. Por ello, el proyecto en sí es una apuesta por la inclusión de una forma práctica

\section{Necesidades educativas de los chicos}

Escola Global ${ }^{2}$, es un colegio internacional de pequeño tamaño que busca un aprendizaje de calidad sustentado en valores que ayuden a los jóvenes a desarrollarse en una sociedad cambiante. Se sigue el currículum británico, con el español y catalán también como lenguas de enseñanza. Las clases son reducidas y priman las metodologías experienciales y activas. "We believe in learning by doing" es la filosofía del centro, que cuenta con una sólida asociación de padres y madres que colabora en una gran parte de los

\footnotetext{
${ }^{2}$ https://escola-global.com
}

proyectos que desarrolla el centro. Aproximadamente un tercio de los niños son españoles, un tercio son alemanes y un tercio provienen de diferentes países europeos, América y otras regiones, permitiendo una comunidad diversa orientada a la formación de ciudadanos en un entorno global. Sin embargo, el español no es la lengua materna para la mayoría de los niños, que encuentran pocas oportunidades en su vida cotidiana fuera del colegio para escribir en este idioma, puesto que en muchas ocasiones su entorno familiar y social se comunica en sus lenguas nativas. Para aquellos para quienes el español es su lengua nativa también esta actividad es muy importante y significativa, en un momento en que las cartas a mano están siendo sustituidas por la comunicación on-line.

Además de este aspecto de trabajar las competencias de lecto-escritura, Escola Global cuenta con unas instalaciones rodeadas de naturaleza, pero se ubica físicamente en un Parque tecnológico, muy cerca de la Universidad y del Hospital Son Espases, con lo que no cuenta con una estructura de barrio o de entorno comunitario como otras escuelas urbanas. Por ello, establecer una comunicación con niños de otros barrios y poblaciones de la isla favorece un trabajo en red que integra a los niños en una comunidad más amplia e incluye a aquellos menores que por su enfermedad tienen menos oportunidades de socialización.

Dentro de este apartado de necesidades de aprendizaje, también es importante destacar el desarrollo sensibilización y empatía, así como la

Paz, B.; Brennan, K. y Verger, S. (2021). Cartas desde nuestro corazón: una experiencia de aprendizajeservicio para favorecer la inclusión de las personas con enfermedades raras. RIDAS, Revista Iberoamericana de Aprendizaje Servicio, 11, 117-127. DOI10.1344/RIDAS2021.11.8 
posibilidad de abrir la visión del mundo de estos chicos hacia espacios de respeto por la diferencia, el conocimiento de las dificultades de grupos poblacionales especiales o de riesgo y el afianzamiento de valores de solidaridad y compromiso social.

Finalmente, se ha de destacar que las actividades desarrolladas en este aprendizaje-servicio priorizan la autogestión del alumnado, con el objetivo de favorecer la creatividad y la resolución de problemas. Así, el apoyo del profesorado es clave para desarrollar iniciativas que en muchos casos surgen del alumnado.

\section{Dimensión del servicio y descripción de las tareas que incluye}

El aspecto vertebrador del servicio en este proyecto de aprendizaje-servicio fue que los alumnos de Escola Global elaboraban cartas para los niños atendidos por INèDITHOS. Sin embargo, la respuesta fue tan buena por parte de los niños de INèDITHOS que se transformó en un proyecto recíproco en el que los niños también elaboraron y enviaron cartas a sus compañeros en Escola Global. En la fecha del primer envío de cartas se hizo entrega además de un paquete con material escolar donado por la escuela a cada niño y que fue empaquetado y organizado también por los niños (febrero de 2019). En mayo, junio y septiembre de 2019 se realizan los siguientes intercambios que incluyen cartas y también dibujos y manualidades. En las tres primeras clases de primaria las cartas se trabajaron conjuntamente el grupo clase. Mientras que de forma individual en el resto de primaria y secundaria.

Como se ha dicho anteriormente, de forma adicional a la redacción de las cartas se llevan a cabo estrategias de recogida de fondos organizadas por el alumnado de secundaria, el centro escolar y la Asociación de padres y madres del centro. Los alumnos de secundaria organizaron un evento donde se vendían alimentos como bocadillos, bizcochos, galletas... preparados o donados por los miembros de la asociación de padres y madres. Adicionalmente, coordinado por el profesor de Arte, se realizó una exhibición de arte inspirado en Asia con una subasta a ciegas de las obras elaboradas por el alumnado La tercera actividad de recaudación consistió en la elaboración y venta de postales realizadas por el alumnado para enviarse unos alumnos a otros para escribirse buenos deseos. La última actividad de recaudación tuvo lugar en el evento de celebración, durante la Feria de Verano organizada en junio por la asociación de padres y madres y el centro escolar. De nuevo, los alumnos de secundaria editaron una revista para vender, prepararon una tómbola y el 'pintacaras'. El profesorado y las familias se organizaron instalando un punto de comida con barbacoa y pizza, bebidas naturales, juegos deportivos, etc. Todo un evento para celebrar la comunidad escolar en su totalidad con INèDITHOS como invitado especial, participando activamente en la celebración del aprendizaje.

Para el desarrollo de estas tareas se ha necesitado la colaboración de la dirección del centro escolar y de la

Paz, B.; Brennan, K. y Verger, S. (2021). Cartas desde nuestro corazón: una experiencia de aprendizajeservicio para favorecer la inclusión de las personas con enfermedades raras. RIDAS, Revista Iberoamericana de Aprendizaje Servicio, 11, 117-127. DOI10.1344/RIDAS2021.11.8 
asociación, voluntarios, docentes, los niños, así como la asociación de padres y madres. Sin embargo, el trabajo en red fue clave y existió desde el inicio del proyecto. La comunicación con el centro escolar fue fluida y de ésta con las familias, bien a través de e-mail o de la plataforma de comunicación educativa Dojo. Por su parte, un grupo de chat creado con los voluntarios de INèDITHOS permitió una comunicación constante a fin de asegurar que todas las partes del proyecto encajaran y fuera lo más satisfactorio posible para todas las partes. Se creó así una colaboración beneficiosa para todos los integrantes en el proyecto (Wrye et al., 2019).

\section{Objetivos educativos, competencias y vinculación curricular}

El currículum británico incorpora la educación para la ciudadanía dada su importancia para la formación integral del alumnado (Crick, 1998), si bien su desarrollo es cuestionable si el centro escolar no impulsa de forma decidida las metodologías que permitan de forma efectiva llevarlo a la práctica (Tonge et al., 2012). Si bien Escola Global sigue el currículum y estructura británica, en cuanto a la lengua española se siguen las directrices del currículum académico balear. Teniendo en cuenta que esta actividad implicó a todo el alumnado a partir de 5 años, los objetivos de este proyecto son distintos según el nivel educativo y el profesorado los adaptó a su grupo clase. En general se enmarcan en el conocimiento de la lengua española, su uso oral y escrito (escuchar, hablar, leer $y$ escribir). Todo el trabajo que se desarrolló en esta actividad se realizó en este idioma a fin de afianzar todos los componentes del uso del lenguaje. Sin embargo, el tipo de proyecto y la población destinataria permitió el trabajo de otras áreas como los valores, el compromiso, la autogestión y la actitud reflexiva. La participación de otras materias como la de Arte también sirvió para acercar al alumnado a la realidad de la expresión artística, tanto en su aspecto creativo como profesional.

\section{Actividades de aprendizaje y de reflexión}

La elaboración de las cartas se realizó en horario escolar, donde los docentes de cada clase estimularon el debate y la reflexión sobre la situación de estos niños. Los niños pasaron varios días componiendo sus cartas. Las clases discutieron las preguntas apropiadas para hacer y analizaron qué preguntas y temas ayudarían a los niños enfermos a sentirse mejor. Esto involucró a los niños con un nuevo nivel de pensamiento y comprensión, ya que a muchos de ellos nunca se les había pedido que pensaran en la situación de otros niños con enfermedades crónicas. De forma práctica, se trabajaron competencias en lengua española, se leyeron cartas en clase, etc. El colectivo docente y el alumnado también aprovecha la situación de que el servicio se desarrolle a lo largo de todo el semestre para hacer constancia de los avances que van desarrollando los niños en su aprendizaje.

Por otro lado, voluntarios de la asociación hicieron una sesión por cada clase, tanto de primaria y como de

Paz, B.; Brennan, K. y Verger, S. (2021). Cartas desde nuestro corazón: una experiencia de aprendizajeservicio para favorecer la inclusión de las personas con enfermedades raras. RIDAS, Revista Iberoamericana de Aprendizaje Servicio, 11, 117-127. DOI10.1344/RIDAS2021.11.8 
secundaria para explicar no sólo el funcionamiento de la entidad, sino también aspectos concretos de lo que son las enfermedades raras y los desafíos que suponen para los niños. También INèDITHOS, al tiempo que traían las cartas al colegio hicieron una presentación mostrando actividades desarrolladas con los niños, algunas de ellas con el material donado, así como mostraron cómo los niños recibían y escribían nuevas cartas. El aprendizaje y la reflexión por tanto se desarrolló también entre los niños atendidos por INèDITHOS, pues pudieron ver como muchas de sus inquietudes eran compartidas por niños que no conocían y eran en muchos casos originarios de otros países.

Se ha de destacar que los niños tienen el protagonismo durante todo el proyecto. La asociación INèDITHOS hace llegar al centro un listado con los niños agrupados por edades e identificados con un código a fin de respetar desde esta fase inicial la confidencialidad y anonimato de los niños. Los niños se agrupan y toman decisiones sobre qué se puede escribir a un niño de su edad, que tiene una enfermedad rara que incide no sólo en la calidad de vida del niño, sino que también, en algunos casos, tiene implicaciones en la esperanza de vida de estos niños. El protagonismo de los niños también es clave para la organización y desarrollo de las actividades de recaudación de fondos y sensibilización descritas antes.

\section{Celebración y difusión}

La asociación de padres ha tenido un papel clave en la celebración y difusión del proyecto. Así, se celebró una feria de fin de curso en que INèDITHOS participó construyéndose una pancarta en que los niños participaron pintando sobre la experiencia. Esta actividad ha servido para dar a conocer más el proyecto y para sensibilizar a la comunidad escolar sobre la situación de los niños con enfermedades crónicas y raras, pero particularmente para celebrar el aprendizaje. Muchos niños y familias se acercaron al Stand de INèDITHOS para conocer más sobre el proyecto.

\section{Evaluación del proyecto}

Se realizó una evaluación a cada grupo clase por el profesorado y además se pasó un cuestionario por parte de INèDITHOS para recibir la valoración del proyecto y evaluar el conocimiento de los escolares sobre las enfermedades raras. Además, se realizaron entrevistas a profesores y voluntarios, que hicieron aportaciones como útiles de cara al desarrollo del proyecto en el curso académico 201920. Una de las mejoras planteadas será la de instalar un Buzón para el intercambio de cartas en el hall del colegio, elaborado en la clase de Arte. Ello será particularmente útil para los alumnos de secundaria, mientras que en primaria el trabajo se seguirá realizando fundamentalmente en el grupo clase.

Se ha de destacar que el proyecto sigue activo de cara a este año próximo a pesar de que una parte del profesorado ha cambiado, lo que da una idea de la forma en que este proyecto está favoreciendo la institucionalización del aprendizaje-servicio en este centro

Paz, B.; Brennan, K. y Verger, S. (2021). Cartas desde nuestro corazón: una experiencia de aprendizajeservicio para favorecer la inclusión de las personas con enfermedades raras. RIDAS, Revista Iberoamericana de Aprendizaje Servicio, 11, 117-127. DOI10.1344/RIDAS2021.11.8 
escolar (Paz-Lourido et al., 2018). Como aspecto de mejora se incluye la utilización de rúbricas y cuestionarios estandarizados para la evaluación del desarrollo e impacto del proyecto (Escofet et al., 2016).

\section{Conclusiones}

Se destaca en este proyecto el alto grado de satisfacción de los niños y las familias atendidas por la asociación INèDITHOS, quienes valoran la dimensión pedagógica, social e inclusiva del proyecto. Para el buen desarrollo del proyecto ha resultado clave el diseño co-participado entre las dos entidades, así como el trabajo en red incluyendo a la asociación de madres y padres del colegio. De cara al futuro, esta colaboración entre INèDITHOS y Escola Global aspira a formar parte de los proyectos aprendizaje-servicio establecidos entre las dos entidades a largo plazo.

\section{Referencias bibliográficas}

Chaves, M. (2012). La pedagogía hospitalaria como alternativa formativa. Educación, 40(21), 59-74. Recuperado de

https://dialnet.unirioja.es/descarga/arti culo/5056878.pdf

Crick, B. (1998). Education for citizenship and the teaching of democracy in schools. Final report, 22 September 1998. London, GB: Qualifications and Curriculum Authority. Recuperado de https://dera.ioe.ac.uk/4385/1/crickrepo rt1998.pdf

Escofet, A., Folgueiras, P., Luna E., y
Palou, B. (2016). Elaboración y

validación de un cuestionario para la validación de proyectos de aprendizajeservicio. Revista Mexicana de Investigación Educativa, 21(70), 929949. Recuperado de http://www.scielo.org.mx/pdf/rmie/v21 n70/1405-6666-rmie-21-70-00929.pdf

Eyler, J., Giles Jr, D. E., \& Braxton, J. (1997). The impact of service-learning on college students. Michigan Journal of Community Service Learning, 4(1), 515. Recuperado de http://hdl.handle.net/2027/spo.323952 $\underline{1.0004 .101}$

Fernández, M., y Grau, C. (2014). Necesidades educativas, asistenciales y sociales especiales de los niños con enfermedades minoritarias: propuestas para una atención interdisciplinar. Revista de Educación Inclusiva, 7(3), 97-124. Recuperado de https://revistaeducacioninclusiva.es/ind ex.php/REI/article/view/134/128

Lake, V. E., \& Jones, I. (2012). Service Learning in the PreK-3 Classroom: The What, Why, and How-to Guide for Every Teacher. Minneapolis, USA: Free Spirit Publishing.

Negre, F. y Verger, S. (2005). Inèdithos: Un proyecto de Pedagogía Hospitalaria dedicado a la mejora de la calidad de vida de niños y jóvenes con enfermedades raras a partir de la intervención de investigación con voluntariado universitario. Aula. Revista de Pedagogía de la Universidad de Salamanca, (23), 107-119.

Ortiz, L., Salom, M. y Verger, S. (2017). Un mundo dentro de un hospital. 
Estudio de caso: Ainhoa. International Journal of Developmental and Educational Psychology, 1(5), 545-553.

Paz-Lourido, B., Negre, F., Verger, S., y de Benito, B. (2018). El aprendizajeservicio en la educación superior: de las experiencias puntuales a la institucionalización. En T. Lleixá, B.

Gros, T. Mauri y J. L. Medina (Coords.), Educación 2018-2020.Retos, tendencias y compromisos (pp. 79-84). Barcelona, España: IRE-UB.

Puig, J. M., Martín, X. y Batlle, R. (2008). Com començar una experiència d'aprenentatge servei. Barcelona, España: Centre promotor d'APS en Catalunya.

Rubio, L. (2009). El aprendizaje en el aprendizaje servicio. En J. M. Puig (Coord.), Aprendizaje Servicio (ApS).

Educación y compromiso cívico (pp. 91106). Barcelona, España: Graó.

Tonge, J., Mycock, A., \& Jeffery, B. (2012). Does citizenship education make young people better-engaged citizens? Political Studies, 60(3), 578602. doi: $10.1111 / \mathrm{j} .1467-$

9248.2011.00931.x

Verger, S., De la Iglesia, B., PazLourido, B., y Negre, F. (2017). Informe de situación sobre la escolarización del alumnado con enfermedades poco frecuentes en las Islas Baleares. En J. Monzón, I. Arostegui y N. Ozerinjauregi (Coords.), Alumnado con enfermedades poco frecuentes y escuela inclusiva (pp.119-148). Barcelona, España: Octaedro.

Wrye, B., Chafin, C., \& Higginbotham,
C. (2019). Creating a Win-Win: Designing and implementing mutually beneficial collaborations between community organizations and academic programs. Education \& Training, 61(5), 605-621. doi: 10.1108/ET-01-20180011 